\title{
A Rare Case Report: Leigh Syndrome
}

\section{Shashikala C Wali*, Mithilesh Shah, Chandani Sheikh, Ashish Singh Parihar, MS Ganachari}

Department of Pharmacy Practice, KLE College of Pharmacy, KLE Academy of Higher Education and Research, Belagavi, Karnataka, INDIA.

\begin{abstract}
It is a case report on a very rare type of Leigh Syndrome (LS) that is adult-onset subacute necrotizing encephalopathy which generally begun during adolescence or early adulthood. It is characterized by degeneration of the CNS, severe failure of oxidative metabolism, vision impairment, impairment of respiratory, heart and kidney function. According to some resources incidence of occurring LS is 1 in 40,000 new born and unusually in adult hood. Characteristics lesion in basal ganglia associated with demyelination. Primary feature includes: subacute relapsing encephalopathy with cerebral and brain-stem signs presenting during infancy. Additional feature includes: basal ganglia lucencies. The prognosis for LS is very poor. Patients lacking mitochondrial complex IV activity and deficiency in pyruvate dehydrogenase have worst prognosis and die within a few years. Patients with partial deficiencies have better prognosis and can live up to 6 to 7 years of age. Some have survived to their mid-teenage years.
\end{abstract}

Key words: Sub-acute necrotizing encephalopathy, Oxidative metabolism, Basal ganglia, Lucencies, Mitochondrial complex, Pyruvate dehydrogenase.

\section{INTRODUCTION}

Leigh syndrome is a rare, classical mitochondrial clinical syndrome, inherited, progressive neurodegenerative condition of the infancy and childhood. ${ }^{1,2}$ There are various synonyms for this condition such as: classical leigh syndrome, leigh necrotizing encephalopathy, necrotising encephalopathy of leigh's Sub-acute Necrotizing Encephalopathy (SNE). ${ }^{3,4}$ Some other divisions of the LS also include: adult-onset subacute necrotizing encephalomyelopathy, infantile necrotizing encephalopathy, X-linked infantile necrotizing encephalopathy. ${ }^{4}$ for the first time it was introduced by a British neuropathologist, Denis Leigh in 1951, in a 7 month baby. The onset of the disease was rapid and result in death over a 6-week period. ${ }^{1}$

LS is characterized by the degeneration of the CNS (i.e. brain, spinal cord and optic nerve). ${ }^{4} \mathrm{LS}$ is also due to the severe failure of oxidative metabolism within the mitochondria of the developing brain owing to a variety of biochemical and molecular defects, including nuclear or mitochondrial DNA mutations. Inheritance can therefore be X-linked recessive, autosomal recessive or maternal depending on the molecular defect. $^{2}$

Onset of symptoms usually begins between the ages of 3 months to 2 years but in some cases clinical presentation do not exhibits for the several years. clinical presentation of LS are associated with progressive neurological deterioration (tremors, muscles spasms that results in slow, stiff movements or absences of tendon reflexes and others symptoms include loss of appetite, diarrhoea, vomiting, dysphagia leading to failure to thrive, irritability, seizure activity, loss of previously acquired motor skills. Further progress of LS arises a symptom such as generalised weakness, lack of muscle tone (hypotonia) and also episodes of lactic acidosis which lead to further complication such as impairment of respiratory, heart and kidney function. Visual problem's such as nystagmus (abnormally rapid eye movement) is also most commonly seen. ${ }^{3,4}$

Respiratory failure is the most common
DOI: 10.5530/ijopp.12.4.59

Address for correspondence: Shashikala C Wali, Department of Pharmacy Practice, KLE College of Pharmacy, KAHER, Belagavi, Karnataka, INDIA.

Phone no: +91-9902944602 Email Id: shashiwali90@gmail. com

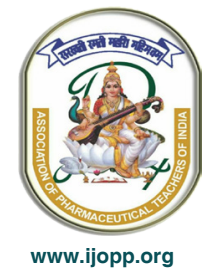


ultimate cause of the death. There are some of the characteristic's lesions in basal ganglia, these are associated with demyelination. ${ }^{3,4}$ currently there is no effective treatment. Some of the classical features of these mitochondrial clinical syndromes (i.e. LS) are: subacute relapsing encephalopathy with cerebellar and brainstem signs presenting during infancy (primary features), basal ganglia lucencies (additional features). ${ }^{5}$

\section{Case Summary}

A 15-year-old male child admitted to paediatric ward who was previously diagnosed as known case of mitochondriopathy and later diagnosed as Leigh syndrome. Patient was semiconscious and not well oriented to time and place. Patients was admitted to the KLE hospital with presenting complaints of difficulties in swallowing both liquid and solid, complaints of increased in generalized body weakness since more than 10 days. On observation abnormal rapid movement of the eye was observed, swelling over the neck was also observed, rapid involuntary movement of the hand was observed, patient was physically as well as mentally unfit for performing any sorts of activities. Currently he was unable to speak and even cannot perform the daily routine activities and was on complete bed bound. Deformity in both upper and lower limb and fingers was observed which was worse than before. The observation of abnormalities has been cited in Figures 1-3.

The child has history of delay in developmental milestones in all the domains, motor and speech. Child was going to school normally till 5 years of age, when he developed generalised weakness, insidious in onset, progressive in nature associated with history of repeated falls. The child started to regress the milestones progressively and was diagnosed as mitochondriopathy and was on treatment.

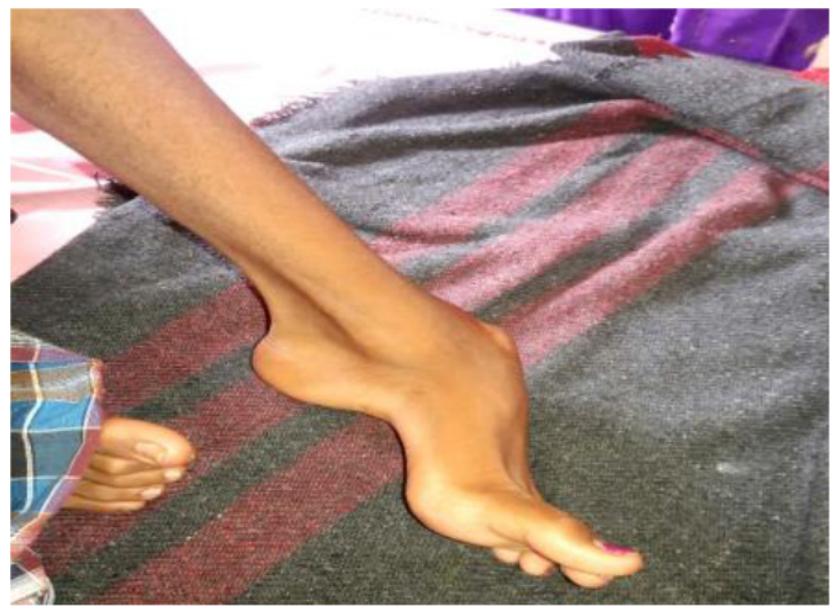

Figure 1: Leigh syndrome with muscular atrophy and deformity of lower limb.

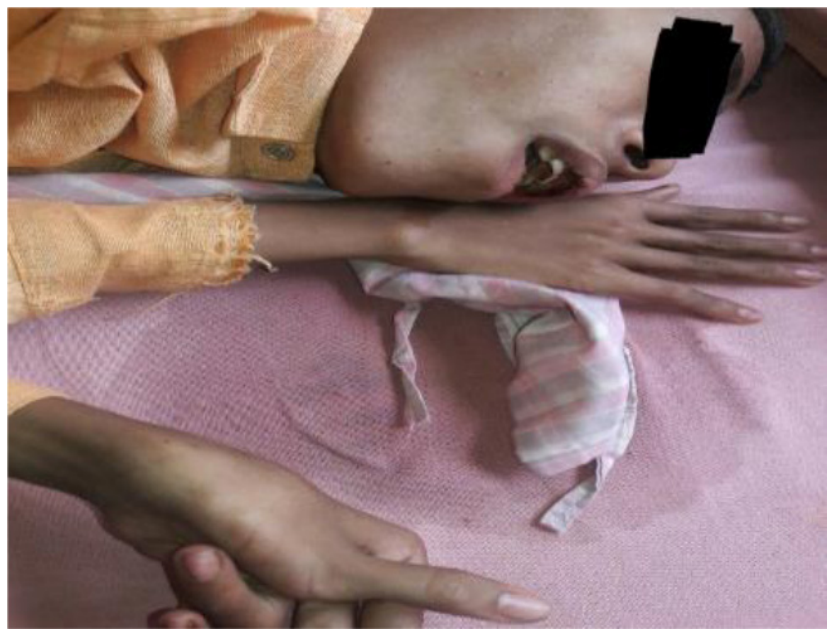

Figure 2: Leigh syndrome muscular atrophy and deformity of lower limb.

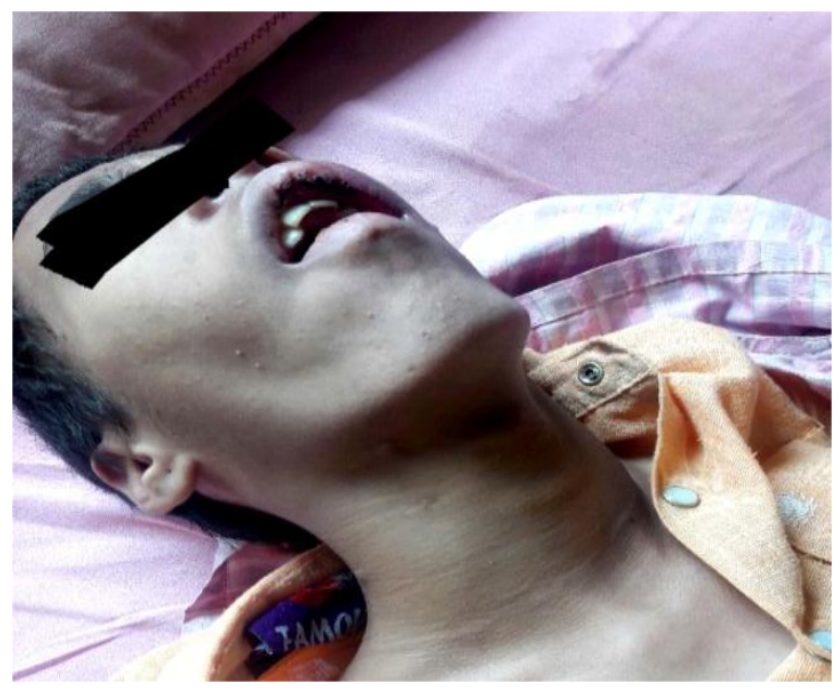

Figure 3: Leigh syndrome with throat swelling and oral deformity, also, injury to lips due to involuntary movement of limbs.

Born of non-consanguineous marriage, had low birth weight, delayed developmental milestones [standing2 years, walking 2.5 years, had weak voice since birth, monosyllables- 3 years, full sentences- 4 years] could not play with other children. No any similar complaints in their siblings and other family members. Child has history of improper and missed vaccination.

Child performed poorly at school, he could not read and write and had learning difficulty. Parents noticed abnormally of gait since the time he started walking, he had easy fatigability. Parents noticed him walking with legs extremely rotated. He had loss of balance after walking moderate distances.

Patient had febrile illness in May 2016, he had high fever for 3 days. After which he developed rapidly progressive altered sensorium, stopped speaking, indicating need, 
became unconscious. He was investigated for the same and was treated for Leigh syndrome (Coenzyme Q, biotin, vitamin b complex, Tab levetiracetam-500mg, Tab clonazepam- $0.5 \mathrm{mg}$, Tab erythromycin- $250 \mathrm{mg}$ ) at the KLE hospital. The patient remain bed bound but regained consciousness, since then he is indicating all the needs with limbs, he is unable to sit on the bed.

The MRI of brain screening which was done on march 2019 shows: Bilateral symmetrical T1 and flair hypointense, T2 hyperintense areas (CSF intensity) noted in the globus pallidus and minimally extending into posterior limb of internal capsule, no obvious restriction on DWI sequence, NO evidence of blooming on SWI sequence, bilateral sylvian fissures appear prominent.

During the admission in KLE hospital recently he was given these treatments: Tab leviteracetam-500 mg, Tab clonazepam- $0.5 \mathrm{mg}$, Tab erythromycin-250 mg, Tab fluconazole-150 mg, Tab trihexyphenidyl-2 mg, Tab risperidone- $0.5 \mathrm{mg}$, vitamin supplement in the form of tablet. Since the treatment of tab levetiracetam and tab clonazepam was continued from more than years, patient developed involuntary movements of both limbs which is vigorous and caused injury to oral parts that is lips. To treat this mild ADR, he was started with another antiparkinson's drug called tab Trihexyphenidyl $\mathrm{HCl}$ $2 \mathrm{mg}$ TD.

\section{DISCUSSION}

P Dinesh et al. ${ }^{6}$ states that LS is a rare disease of CNS and chance of occurring is 1 in 40,000 new born and certain population of Saguenay Lac-Saint-Jean region of Quebec, Canada 1 in 2,000 and age of start of symptoms usually less than 2 years and also may presents in childhood but unusually in adulthood and here $\mathrm{I}$ it is a case of onset of LS at age of 13 year. Among different studies patient suffering from LS, the family history was found positive in only some of the study. As of our case in some of the other case study also the onset of the LS symptoms was found in later age.

Arii $\mathrm{J}$ et al. ${ }^{7}$ concluded that biochemical or genetic abnormalities was found in less than 35\% to $60 \%$ of patients with LS in the same way in my case also biochemical and genetic abnormalities has not any significant role for development in LS. Some of the study also described that some of the LS patient have survived to their mid-teenage years which is very uncommon and, in this case, it has happened.

\section{CONCLUSION}

It is a case report on a very rare type of Leigh Syndrome (LS) i.e. adult-onset subacute necrotizing encephalopathy which generally begun during adolescence or early adulthood. From this case report we can illustrate that sometimes biochemical and genetic abnormalities is not playing significant role in development in LS. It is characterized by degeneration of the CNS, severe failure of oxidative metabolism, vision impairment, impairment of respiratory, heart and kidney function. LS is very common in age below 5 years but rarely sometimes it is seen in mid-teenage groups.

\section{ACKNOWLEDGMENT}

We would like to thank Head of the Department of Paediatrics KLE's Dr. Prabhakar Kore Hospital and medical research centre Belagavi and Principle of KLE College of Pharmacy, Belagavi.

\section{CONFLICT OF INTEREST}

The authors declare no conflict of interest.

\section{ABBREVIATIONS}

LS: Leigh Syndrome, SNE: Sub-acute necrotizing encephalopathy, CNS: Central nervous system.

\section{SUMMARY}

Presented case report is prepared on rare Leigh syndrome.

\section{REFERENCES}

1. Shrikhande DY, Kalakoti $\mathrm{P}$, Syad $\mathrm{AMM}$, et al. A rare mitochondrial disorder: Leigh syndrome- a case report. Italian Journal of Paediatrics. 2010;36(62):1-5.

2. Leigh syndrome, Greenfield's neuropathology, CRC Press, $9^{\text {th }}$ edition. 2015;1:527-35.

3. Stacpoole PW. Leigh Syndrome. National organization for rare diseases. (NORD). Available from: https://rarediseases.org/rare-diseases/leighsyndrome/ Accessed on 28 May 2019.[Internet]

4. Lake NJ, Bird MJ, Isohanni P, et al. Leigh syndrome: Neuropathology and

5. Leigh's Disease Information Page. National institute of neurological disorder and stroke. Available from: https://www.ninds.nih.gov/Disorders/All-Disorders/ Leighs-Disease-Information-Page Accessed on 28 May 2019.[Internet]

6. Dinesh P, Madan RM, Gita S. Leigh syndrome: an unusual rare case report. Inte J Sci Stud. 2014;2(2):93-6.

7. Arii J, Taanabe Y. Leigh syndrome: Serial MR imaging and clinical follow-up. AJNR Am J Neuroradiol. 2000;21(8):1502-9. 